\title{
Topography and Grounding in a Simple Bottom Layer Model
}

\author{
KEVIN SPEER \\ Laboratoire de Physique des Océans, Institut Fransais de Recherche pour l'Exploitation de la Mer, \\ Plouzané, France \\ ELI TZIPERMAN
}

Weizmam Institute of Science, Rehovot, Israel

YIZHAK FELIKS

Institute for Biological Research, Nes-Ziona, Israel

\begin{abstract}
A reduced-gravity model for a dense bottom layer under a motionless deep layer is used to investigate bottom layer flow with topography. The two competing effects in the model are topography, which introduces a topographic $\beta$ effect, and strong layer thickness variation, which allows for the possibility of zero-layer thickness or grounding. The goal is to arrive at a simple, observationally relevant characterization of the model which can help to distinguish different dynamical mechanisms operating in the ocean. Several regimes are identified, including situations for which the layer depth goes to zero with and without topography. Ways of distinguishing observationally between competing effects are suggested. The study is motivated by the observed shift of Antarctic Bottom Water in the western North Atlantic Ocean from the west to the east; this phenomenon is used to discriminate key elements of the model.
\end{abstract}

\section{INTRODUCTION}

Water made dense within Antarctic embayments spreads north and entrains water lying above it, especially in regions where it accelerates, such as the Antarctic continental slope and in passages between basins. In a diluted form then, it is the source of the densest water to almost every basin in the World Ocean [Mantyla and Reid, 1983]. Going beyond a general outline of the sources of dense water to a basin to an explanation of the detailed structure of the flow requires a theoretical framework, and the most successful one is certainly the Stommel and Arons [1960] model [Warren, 1981]. Beginning with specified sources and postulating a uniform interior upwelling, the interior and boundary current flow pattern and strength may be calculated. Given the success of the model what is to be gained by further complication?

The fact is that it becomes difficult to justify a flat-bottom flat-interface model, if one wishes to study a bottom water mass, as opposed to the entire deep water layer below the thermocline. Basin geometry modifies the flow through a nonzero vertical velocity at the bottom, and also through a variable basin area with depth, which modulates the upwelling for a given mass source to the basin. Below the mostly flat isopycnals at mid-depth and low- and midlatitudes, isopycnal surfaces near the transition from deep to bottom water can have vertical excursions as large as the thickness of the bottom water layer itsell.

A number of interesting extensions of the Stommel and Arons model have been performed, including permeable eastern boundary [Warren, 1982; Waren and Speer, 1991], vertical structure [Tziperman, 1986; Speer and Tziperman,

Copyright 1993 by the American Geophysical Union.

Paper number $92 \mathrm{JC} 03018$.

$0148-(0227 / 93 / 92 \mathrm{JC}-03018 \$ 05.00$
1990], topographic $\beta$ effect [Waren and Owens, 1985], topographically induced closed potential vorticity contours [Straub and Rhines, 1990; Kawase and Straub, 1991], hypsometry [Rhines and McCready, 1989; Speer and Tziperman, 1990], spin-up and nonuniform mixing [Kawase, 1987], and large interface depth excursions with grounding [Speer and McCartney, 1992]. All of these extensions appear to have a region or basin where they are particularly relevant. The difficulty of combining several of these extensions with boundary currents into an analytic model of a closed basin has led us to formulate a numerical one. The goal is to illustrate aspects of the flow or layer depth variations which can be fruitfully observed to learn something of the dynamics, and help to decide which processes are of central importance in the ocean.

This problem is about the large-scale distribution of mass in a basin. Thus the depth of the layer's interface must be calculated everywhere, and not specified as, e.g., a background state or an eastern boundary condition. The initial condition is inllow at the source and zero thickness elsewhere in the basin, so this might be called a basin "fill-up" problem, as opposed to the usual "spin-up"; however, we are mainly interested in the steady state limit.

In large-scale ocean dynamics problems, one is used to thinking of characteristics or planetary waves carrying the eastern boundary condition on layer thickness westward into the interior. When there is zero-layer thickness, some other mechanism must first distribute the water around the basin. In our damped, linear momentum equations a bottom Ekman layer flow allows water to cross isobars and isobaths and fill the basin. The basic fat-bottom response is that as the basin fills and the flow aligns itself with isobars (being dominantly geostrophic), upwelling forces interior water columns to gain potential vorticity $q=f / h$ and they move poleward in response. 
With bottom topography the steady state distribution of a and its relation to interior flow and boundary currents is more complex. Several model solutions will be shown; for three of these the usual western boundary current flowing north from a source at the southern boundary of the basin switches sides, and moves north along the eastern boundary. Nevertheless there are differences between the potential vorticity distribution and the location of boundary currents in the solutions which may help to identify and distinguish dynamics that result in similar-appearing structures in the ocean. Key identifying structures include the location of the grounding line where thickness is zero, and the presence or absence of a low-latitude western boundary current.

\section{MODEL}

The model describes the motion and thickness of a bottom layer under a resting deep layer, and above a variable seafloor topography. The bottom layer is fed by a mass source $S=4 \times 10^{6} \mathrm{~m}^{3} \mathrm{~s}^{-1}$ at the southern boundary, and this mass leaves the layer by upwelling across the interface between bottom and deep water. The dynamics are geostrophic to lowest order, with weak bottom friction. Similar systems with linear, nearly geostrophic momentum equations have been used recently in a number of studies. Salmon [1986] and Colin de Verdiere [1988] used the planetary geostrophic momentum equations with a full density equation in a continuously stratified model. Rhines [1989] investigated the same $1 \frac{1}{2}$ layer model as here, but with a different form of dissipation and different geometry (see below).

The model geometry is a rectangular basin meant to represent a region $30^{\circ}$ wide extending from $2^{\circ} \mathrm{N}$ to $52^{\circ} \mathrm{N}$. The equatorial $\beta$ plane approximation is used, with the Coriolis parameter $f=\beta y$. The hydrostatic relation may be used to write the pressure in terms of interface height; then the momentum and mass conservation equations are

$$
\begin{gathered}
r u / h-f v=-g^{\prime}(h+b)_{x} \\
r v / h+f u=-g^{\prime}(h+b)_{y} \\
u_{x}+v_{y}+w_{z}=0,
\end{gathered}
$$

where $x, y, u, v$ are the zonal and meridional directions and speeds, positive east and north, $h$ is the layer thickness, $b$ is the bottom elevation representing topography, $g^{\prime}=g \Delta \rho / \rho$, where $g$ is gravity, $\Delta \rho$ is the density difference between the bottom layer and the layer above it, and $\rho$ is the bottom layer density. The reduced gravity used here varied between 0.1 and $0.2 \mathrm{~cm} \mathrm{~s}^{-2}$. Increasing $g^{\prime}$ has the effect of stiffening the interface and reducing its slope for a given current. The absence of time derivatives in (1)-(2) filters out Kelvin and other gravity waves.

The form of friction chosen here is a result of vertically integrating a vertical dissipation term like $\nu u_{z z}$, and setting the bottom stress to be proportional to the velocity. This form of friction guarantees that as the thickness vanishes, so do the horizontal velocities. Furthermore, the strong damping of horizontal velocity near outcrops implies that nonlinearities in the momentum equations are negligible there. The resulting frictional flow essentially amounts to distributing the bottom Ekman layer flow throughout the layer, except that the $f$ dependence of the Ekman layer thickness $(\nu / 2 f)^{1 / 2}$ has been ignored, thus avoiding an Ekman layer thicker than the bottom layer near the equator. The coefficient $r$ varies between 0.05 and $0.3 \mathrm{~cm} \mathrm{~s}^{-1}$ in all runs. The size of the frictional term relative to the Coriolis term is represented by the scale $r / f H$. With typical interior values $r=0.1 \mathrm{~cm} \mathrm{~s}^{-1}$, $f=10^{-4} \mathrm{~s}^{-1}$, and $H=10^{5} \mathrm{~cm}$, this scale is $O\left(10^{-2}\right)$; thus we are in a nearly geostrophic regime.

Linearizing about a mean layer thickness $H$ leads to the usual western, northern, and southern boundary layers at walls, of width $r / \beta H$ in the west and wider $(r L / \beta H)^{1 / 2}$ in the north and south, where $L$ is the basin width. Attention was given to the problem of resolving these boundary layers when their width varies owing to strong layer thickness variations. In practice this meant increasing $r$ for those runs in which the layer thickness became especially large. A two grid-point wide boundary layer was found to be adequate resolution; some test runs with a somewhat higher resolution (more grid points) showed no apparent special behavior or structure.

The strength of the dissipation can also be measured by the spin-down time $H / r$. The spin-down time varies with the thickness scale and can be as short as 1 day and as long as 1 month, depending on location. Typically, it is 2 weeks, an order of magnitude longer than the inertial period. Previous numerical studies of a bottom layer isolated the topography from the side boundary layers, thus allowing interior friction to be reduced; here there is no such distinction, and the "sides" of the bottom layer evolve with the flow (as do the boundary layer widths). Friction is automatically reduced in the interior, where thickness is greatest.

The mass equation (3) can be integrated vertically to the top of the layer, where mixing gives rise to an entrainment into the upper layer, of strength $w_{e}$ :

$$
h_{t}+\nabla \cdot(\mathbf{u} h)=-w_{c} .
$$

The equations for velocity and thickness are solved numerically on a staggered grid (see appendix for details of the method). Starting from an initial $h$ field the velocity can be found using the momentum equations (1)-(2), and then $h$ at the next time step is calculated from (4) using an AdamsBashforth time step method. This procedure is repeated with a time step of approximately one-half day until the fields have reached a steady state.

Combining (1)-(3) gives the vorticity equation

$$
(r v / h)_{x}-(r u / h)_{y}+\beta v=f w_{z} .
$$

Integrating this equation vertically, using (4), and defining $q=f / h$ give the potential vorticity equation used in the following discussion,

$$
\frac{d \ln q}{d t}=w_{e} / h-r f^{-1}\left[(v / h)_{x}-(u / h)_{y}\right],
$$

where $d / d t$ is the total derivative following the flow.

One can also write the integrated vorticity (5) and mass (3) equations as

$$
h_{t}=\beta h P_{x} l f^{2}+w_{E}-w_{b}-w_{c}
$$

where the frictional flow divergence or pumping $w_{E}=-r \zeta / f$ $-r \beta u l f^{2}, P=g^{\prime}(h+b)$, and $w_{b}=-J(P, b) / f$ with $J$ the Jacobian. At steady state there is a balance between the terms on the right-hand side: divergence between isobars, 
pumping, upwelling, and geostrophically generated vertical motion at the bottom.

The same basic model with a somewhat different form of dissipation has been described by Rhines [1989], who added a background barotropic flow and investigated flow over isolated topography. To see the relation to his system, (1)-(3) can be written in terms of interface height $\eta=h+b$ :

$$
\eta_{t}+c^{x} \eta_{x}+c^{y} \eta_{y}=-w_{e}+\nabla \cdot(k \nabla \eta)
$$

where

$$
\begin{gathered}
c^{*}=-\frac{g^{\prime} f\left(1+2 R^{2} / F^{2}\right)}{F^{2}} b_{y^{\prime}}-\frac{\beta g^{\prime} h}{F^{2}} \\
c^{y}=\frac{g^{\prime} f\left(1+2 R^{2} / F^{2}\right)}{F^{2}} b_{x} \\
k=\frac{r g^{\prime}}{F^{2}} \\
R=r / h \\
F^{2}=f^{2}+\frac{r^{2}}{h^{2}}
\end{gathered}
$$

Dropping all dissipation terms $O\left(r, r^{2}, r^{3}\right)$ gives Rhines' [1989] equation (3.1) for the interface evolution without background flow and dissipation. The bottom friction form of dissipation used here gives rise to the familiar replacement of $f$ by $F$. The diffusion coefficient $k$ is spatially variable, unlike the simpler constant used by Rhines [1989]. Expanding the divergence of the diffusive flux produces a small pseudovelocity term $\left(2 \beta f k / F^{2}\right)$ in $c^{y}$, tending to carry interface displacements northward, plus an additional interaction between thickness and pressure resulting from vertical velocities at the top and bottom forced by downgradient frictional flow. Some of the ageostrophic flow divergence is related to a divergence in the pressure gradient $\left(\nabla^{2} \eta\right)$, thus leading to an effective diffusion of interface displacement in (8). A further divergence in frictional flow results from changes in $F$. Expanding the diffusion term gives

$$
\nabla \cdot(k \nabla \eta)=k \nabla^{2} \eta-\frac{2 \beta f k}{F^{2}} \eta_{y}+\frac{2 R^{2} k}{h F^{2}}(\nabla h \cdot \nabla \eta) .
$$

Though one cannot strictly speak of characteristic velocity for the system with dissipation, it is clear that friction "slows down" the propagation of information by reducing the effective velocities $c^{x}$ and $c^{y}$. The degree of reduction is approximately $1-f^{2} / F^{2}$, which is typically less than $10 \%$. On the other hand, friction augments the spreading along topographically guided characteristics by the term $2 R^{2} / F^{2}$.

Rhines' [1989] purpose was to expose the range of dynamics found in this apparently simple system. The main differences between Rhines' [1989] study and ours is our emphasis on the structure of a bottom layer in a basin fed by a mass source, that is, for a basin which satisfies an integrated mass balance, and also on the ultimate interaction between topography and layer thickness resulting from grounding.

On the equator, $f$ is zero, and ( 8 ) becomes

$$
\eta_{l}-\frac{\beta g^{\prime} h^{3}}{r^{2}} \eta_{x}=-w_{e}+\nabla \cdot\left(\frac{g^{\prime} h^{2}}{r} \nabla \eta\right) .
$$

The westward penetration speed has reached its maximum constrained by damping. This behavior mimicks long equatorial waves, not present in the model, and allows the domain to be extended across the equator. Runs with cross-equatorial flow were made, but are not the focus of the present study.

Before presenting the numerical solutions, it is useful to consider some simple topographic forms for which there are analytic solutions, to examine the consequences topography has on two key aspects of bottom layer structure: the zero-thickness curve or grounding line, and the direction of the interior flow. If we ignore friction for the moment $(r=$ 0 ), and restrict to $b=b(y)$, some progress can be made on the structure of $h$, and the location of the grounding curve where $h=0$.

With topography of height $b(y)$ above a level surface, and a layer thickness $h(x, y)$, the interface height, whose gradient is proportional to the horizontal pressure gradient, is $\eta=h+b$, and

$$
\begin{gathered}
u=-\frac{g^{\prime}}{f}(h+b)_{y} \\
v=\frac{g^{\prime}}{f} h_{x}
\end{gathered}
$$

Substituting in (4) and rearranging,

$$
\left(\beta+\frac{b_{y} f}{h}\right) v=\beta_{e f f} v=\frac{f w_{e}}{h}
$$

in the new vorticity equation. The topographic term in the effective planetary vorticity gradient may augment or diminish planetary $\beta$, depending on the sign of $b_{y}$. Higher order dynamics (friction or vorticity advection) are needed wherever $h \beta_{e f f}$ goes to zero, to eliminate infinite velocity. Huang [1984] has derived the boundary layer structure of an isopycnal outcrop, the surface analogue of grounding, and $\mathrm{Cush}$ man-Roisin [1986] shows how to include the first effects of vorticity advection at such a front on a mid-latitude $\beta$ plane; a frictional balance will be discussed in the next section.

Substituting $\vec{h}=h+f b_{y} / \beta$ gives the standard flat-bottom equation

$$
\left(\frac{\bar{h}^{2}}{2}\right)_{x}=\frac{f^{2} w_{e}}{\beta g^{\prime}}
$$

which can be integrated from the eastern or western boundary, $x_{0}$, where $\widetilde{h}$ nust be specified.

The equation for the grounding curve on which $h=0$ is, with $h\left(x_{0}\right)=h_{0}$,

$$
x-x_{0}=-\frac{\beta g h_{0}}{2 f^{2} w}\left(h_{0}+\frac{2 f b_{y}}{\beta}\right) .
$$

If this equation is scaled, it becomes

$$
\left(x-x_{0}\right) y^{2}=-\frac{\lambda}{w}\left(1+2 y b_{y}\right)
$$




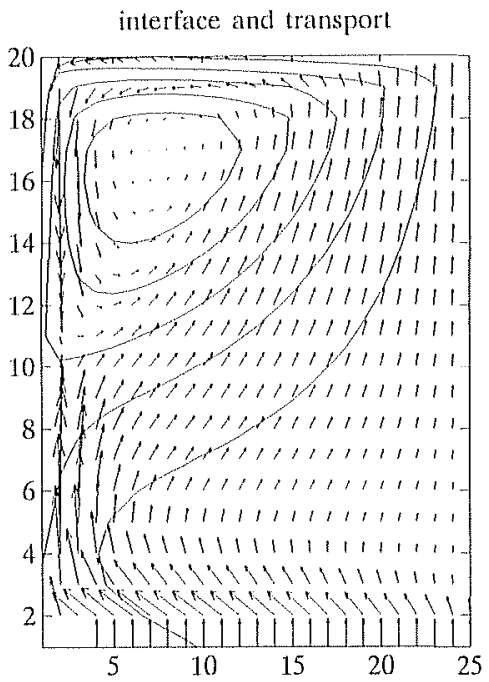

interface $\min =137 \max =652$

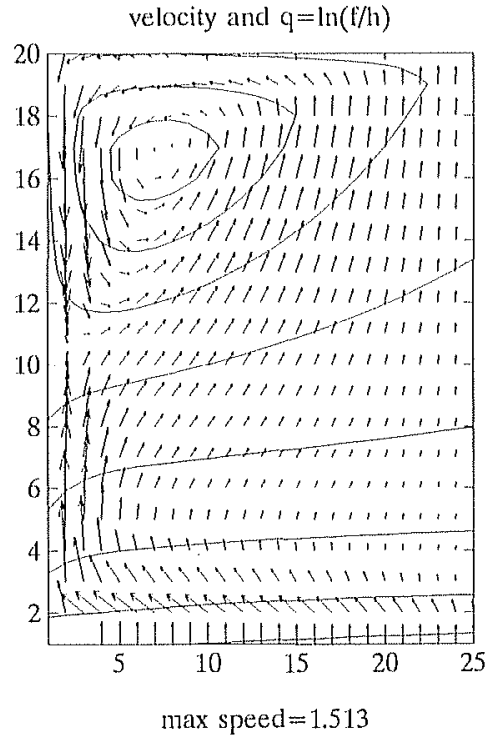

Fig. 1. Flat-bottom reference case, run a (see Table 1). The domain is from $2^{\circ} \mathrm{N}(y$ gridpoint $=1)$ to $52^{\circ} \mathrm{N}(y$ gridpoint $=20$ ), and $30^{\circ}$ wide $(x$ gridpoint from 1 to 25). The interface height is in meters, and speed is in centimeters per second; the reduced gravity $g^{\prime}=0.1 \mathrm{~cm} \mathrm{~s}^{-1}$, and $r=0.2 \mathrm{~cm} \mathrm{~s}^{-1}$. using $x, y \sim L, w \sim S / 2 L^{2}$, and $b \sim h_{0}$. The parameter $\lambda=g^{\prime} h_{0} / \beta S L$ represents the ratio of the adjustment time due to long planetary waves and the residence time in the basin [Speer and McCartney, 1992].

The westward extent of the bottom layer $x-x_{0}$ increases when the bottom shoals poleward $\left(b_{y}>0\right)$ as the background planetary vorticity gradient is reinforced (westward propagation speeds in (8) are greater). The reverse occurs for deepening, and the layer grounds farther east. If the deepening is so strong as to change the sign of $\beta_{c f f}$, the interior meridional flow reverses (12) and eastern boundary currents are appended to balance mass in the basin. Strong basinscale deepening means similarly strong layer thickness variation, which in turn modifies $\beta_{\text {eff }}$. Indeed, a thickness change may force $\beta_{\text {eff }}$ to change sign somewhere up the basin, causing a retransposition of boundary currents and associated frictional flow across the interior (see below and Figure 3).

\section{RESULTS}

\subsection{Flat-Bottom Cases}

Two flat-bottom cases may be used as references (Figures 1 and 2). There are a number of possibilities when it comes to choosing the exact location of the southern source. It was decided to spread this source across the entire southern boundary to avoid biasing the solution toward the appearance of either a western or an eastern boundary current. A table of runs summarizes two key characteristics of the
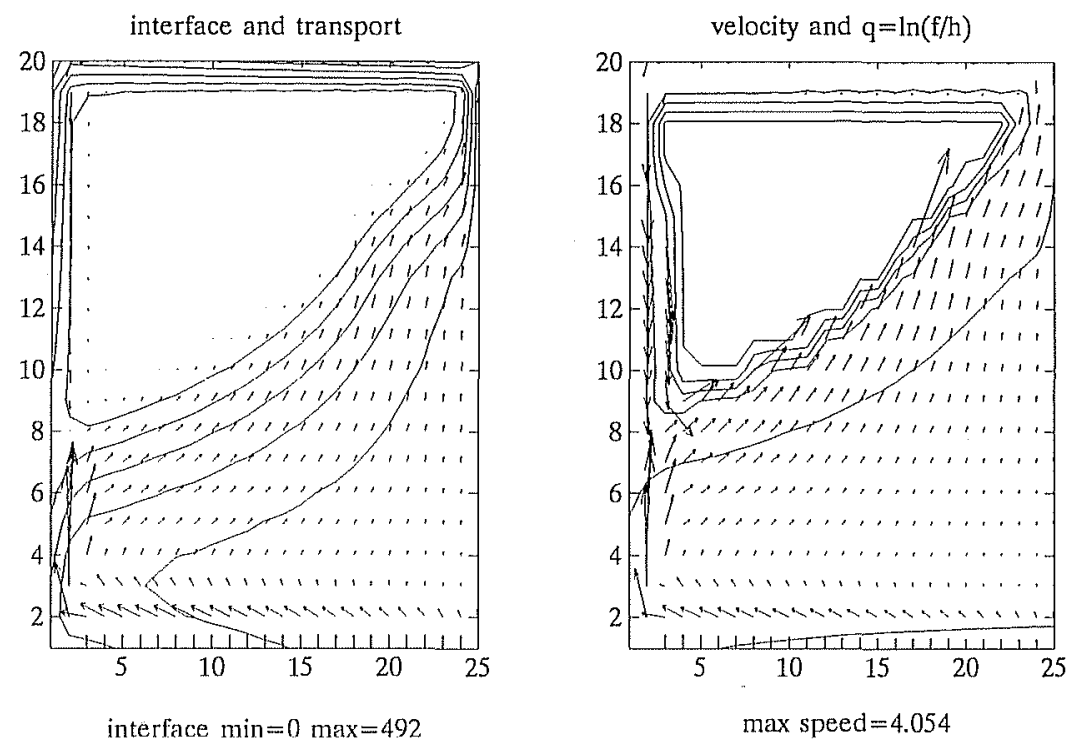

Fig. 2. Grounding case, run $b$ ( $w^{\prime}{ }^{\prime}$ greater than source divided by the area of the basin). The reduced gravity $g^{\prime}=0.1$ $\mathrm{cm} \mathrm{s} \mathrm{s}^{-1}$, and $r=0.05 \mathrm{cn} \mathrm{s}^{-1}$. 
TABLE 1. Summary of Model Runs Used in This Study

\begin{tabular}{|c|c|c|c|c|}
\hline Run & Topography & Grounding & $\begin{array}{c}\text { Low-latitude } \\
\text { WBC }\end{array}$ & Figure \\
\hline $\mathbf{a}$ & flat & no & yes & 1 \\
\hline $\mathrm{b}$ & fat & yes, NW corner & yes & 2 \\
\hline $\mathrm{c}$ & Jinear slope in southern half & no & no & 3 \\
\hline $\mathrm{d}$ & bowl shape & no & no & 4 \\
\hline $\mathrm{e}$ & bowl shape & yes, SW corner & no & 5 \\
\hline
\end{tabular}

model solutions presented here, which are the occurrence of grounding and the presence of a low-latitude western boundary current (Table 1).

First consider a basin with a flat bottom fed by a source weak enough to avoid grounding (Figure 1; Table 1, run $a$ ). Western, northern, and southern boundary currents are allowed in the flat case and all are evident, including the westward thickening of the northern and southern boundary layers, as expected from a simple boundary layer analysis. The flow is mainly parallel to the interface $h+b$, although there is a substantial cross-isobar component owing to friction.

For comparison the velocity is also shown, and the relatively stronger values in the northwest corner where the layer thins hints at nonlinear effects to come: the amplification of streching by thinning. This happens because, from (8), when $h$ decreases, the characteristic velocity $c^{x}$ slows, building up stronger interface gradients. The potential vorticity field $q=f / h$ is substantially perturbed from the background field $f / h_{0}$, as a result of strong layer thickness variations.

By turning up the upwelling velocity to a value greater than the source divided by the basin area, the interface is driven into the ground (Figure 2; Table 1, run $b$ ). The "outcrop area" of the bottom, where the bottom is in contact with the motionless overlying water, grows until the balance

$$
w_{e}(\text { basin area }- \text { outcrop area })=\text { source }
$$

is obtained. (Such a steady balance would be modified to an integral over the area of the interface if the upwelling were a function of flow and thickness). The outcrop area is ringed by an isolated current which transports interior water back toward the source, and this water is re-injected in a band along the interior grounding curve $h=0$. In the model the balance of forces in this isolated boundary current is dominantly that the downstream pressure gradient equals drag, the drag being due to flow balanced geostrophically by a cross-stream pressure gradient.

To complete the description of the model, the structure of $h$ next to the grounding curve may be investigated by dropping small $y$ derivatives from the steady, flat-bottomed version of (8):

$$
\frac{-\beta g^{\prime} h}{F^{2}} h_{x}=\left(\frac{r g^{\prime}}{F^{2}} h_{x}\right)_{x}-w_{e} .
$$

To simplify the analysis, $F$ is taken to be constant where it appears in the $\beta$ term, which overemphasizes the eflect of $\beta$ only when $h=O(r / f)=10 \mathrm{~m}$, by which point the other terms dominate anyway. It is important, however, to relain variable diffusivity ( $k$ in $(8)$ ). (Note that damping of the form
- ru leads to a constant $F$ but variable diffusivity $k=$ $g^{\prime} r h /\left(f^{2}+r^{2}\right)=g^{\prime} r h / F^{2}$.) Integrating and rearranging gives, using the condition $h=0$ at $x=0$,

$$
h_{x}=\frac{-\beta h^{2}}{2 r}+\frac{f^{2} w_{e} x}{2 r g^{\prime}}+\frac{r w_{e} x}{2 g^{\prime} h^{2}} .
$$

From (17), when $h$ is very small the role of rotation is eliminated, leaving

$$
h^{2} h_{x}=\frac{r w_{e}}{2 g^{\prime}} x
$$

with solution $h=\left(3 r w_{e} x^{2} / 2 g^{\prime}\right)^{1 / 3}$ as the structure nearest the grounding curve. From (1), with $f=0$, the flow $u=$ $-g^{\prime} h h_{x} / r=-w_{e} x / h$ is just the frictional flow required to satisfy continuity. Near the grounding curve the advection of the interface by the frictional flow is crucial, as it tends to raise an interface flattened by diffusion and upwelling.

At larger scales, a sequence of consistent balances obtains between the $\beta, w_{e}$, and $r$ terms, until at the largest scales the $\beta$ term balances stretching. Without mixing $\left(w_{c}=0\right)$ and the associated stretching, a balance between the remaining terms would lead to a hyperbolic tangent solution more relevant during spin-up.

The velocity field shows a few spuriously large values near the grounding curve (though transport is well behaved). Velocity increases somewhat toward the grounding curve in the interior as the layer thins, until friction eventually shuts off the flow. The potential vorticity field is completely dominated by the existence of the outcrop. With these two flat-bottom cases with and without grounding as a reference, the influence of topography on the solution may be examined.

\subsection{Adding Botlom Topography}

Although the initial filling-up stage of the problem is not the main focus here, it is helpful as an introduction to discuss the time evolution for the case in which dense water enters the basin in the south and encounters a downward sloping bottom. Gravity pushes it down the slope initially, but this force is rapidly balanced by the Coriolis force. Were it not for a third force, bottom drag, the flow would rapidly be constraned to follow an isobath. Drag slows the flow, reducing the Coriolis force component, and allows gravity to continue to push the fluid downhill. Finally, drag, gravity, and the Coriolis fores all balance at some angle to the isobaths. This situation is common to plume models [Smith, 1975], but whereas they are meant to represent the entrainment part of the plume's trajectory, we assume that this stage is already over with and that the plume has begun its detrainment stage [Speer and Tziperman, 1990]. The loss of 

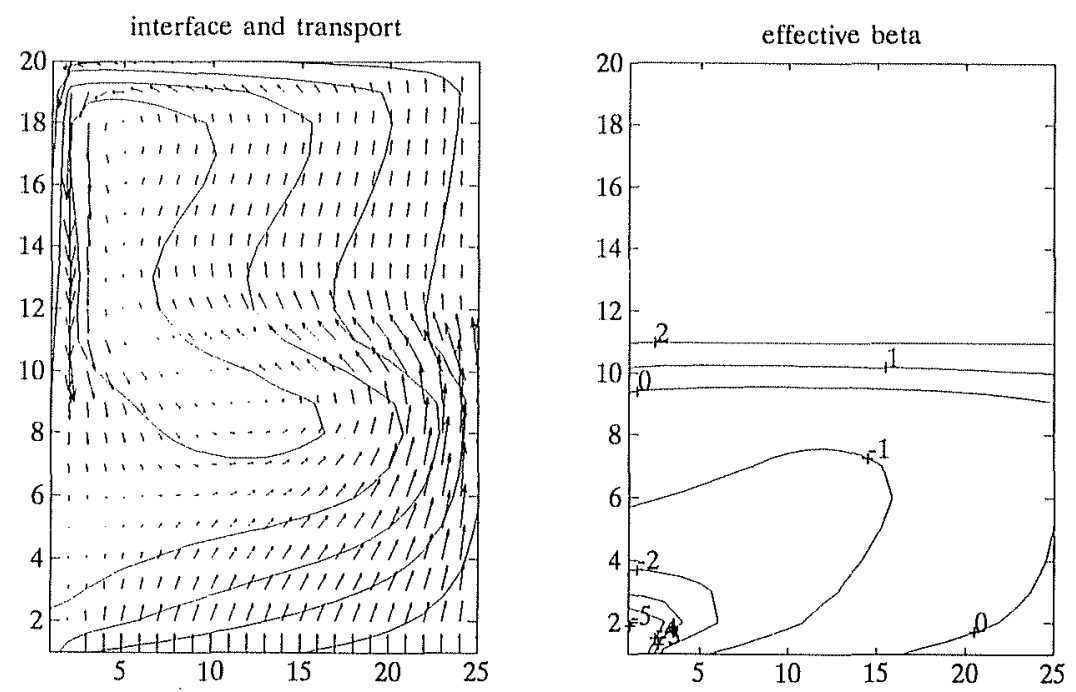

interface $\min =-111 \max =157$
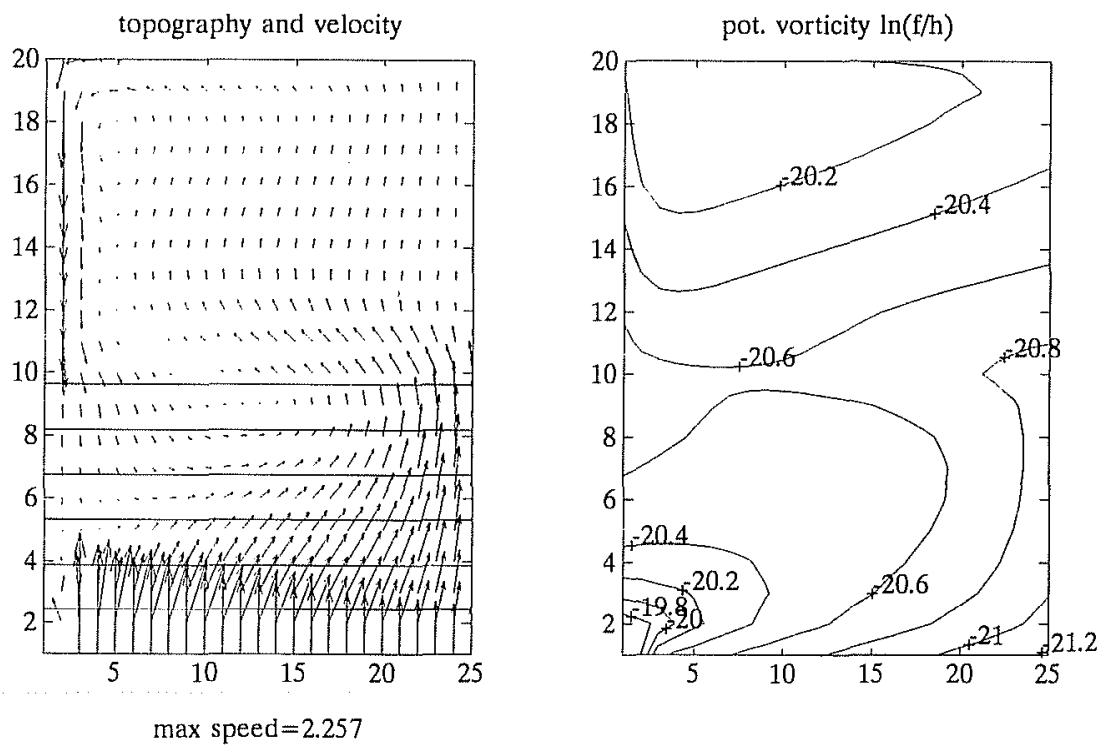

Fig. 3. Sloping bottom case, run $c$ (slope only in the southern half of the basin). The thickness at the northern boundary in the absence of motion is $1000 \mathrm{~m}$; the mean thickness is $470 \mathrm{~m}$. The reduced gravity $g^{\prime}=0.2 \mathrm{~cm} \mathrm{~s}^{-1}$, and $r=0.17 \mathrm{~cm} \mathrm{~s}^{-1}$. Effective beta in units of $10^{-13} \mathrm{~cm}^{-1} \mathrm{~s}^{-1}$.

water by the plume is tiny at first, since the upwelling is uniform and the area of the plume is small. Eventually, some detrainment does occur during the fill-up stage because the plume spreads out enough under the influence of friction that even the weak upwelling causes it to lose some water. A serious fault in the model's simulation of the initial plume, though, is the absence of inertia. The advection of momentum is probably important to the structure of the plume, as Nof et al.'s [1990] density current solutions have shown. On the other hand, once a large-scale bottom layer flow is established, we expect inertia to be small compared to friction, the pressure gradient, and the Coriolis force.

Consider now the solution when a bottom slope is introduced into the model and the first effect of topography is examined, which is the modification of the background potential vorticity gradient or topographic $\beta$ effect. A bottom deepening from south to north in the southern part of the western North Atlantic has been suggested as an explanation for the transposition of northward flow from the western side of the basin to the eastern side [Warren, 1981] and the actual oceanic slope seems to be strong enough for such an effect to be significant. Is this mechanism for eastward intensification stronger than grounding? The next three cases with topography are presented to show how one might determine the relative importance of the effects by examining potential vorticity and the structure of the flow in the bottom layer.

In the presence of a bottom slope, the model flow does indeed transpose (Figure 3; Table 1, run $c$ ) and an eastern boundary current is formed over the southern half of the basin where the slope exists. The circulation in the northern part of the basin is just that of the usual flat bottom solution (Figure 1). To demonstrate the importance of topographic $\beta$ the effective beta $\left(\beta+f b_{y} / h\right)$ is also plotted, and it is negative over almost all of the slope. 

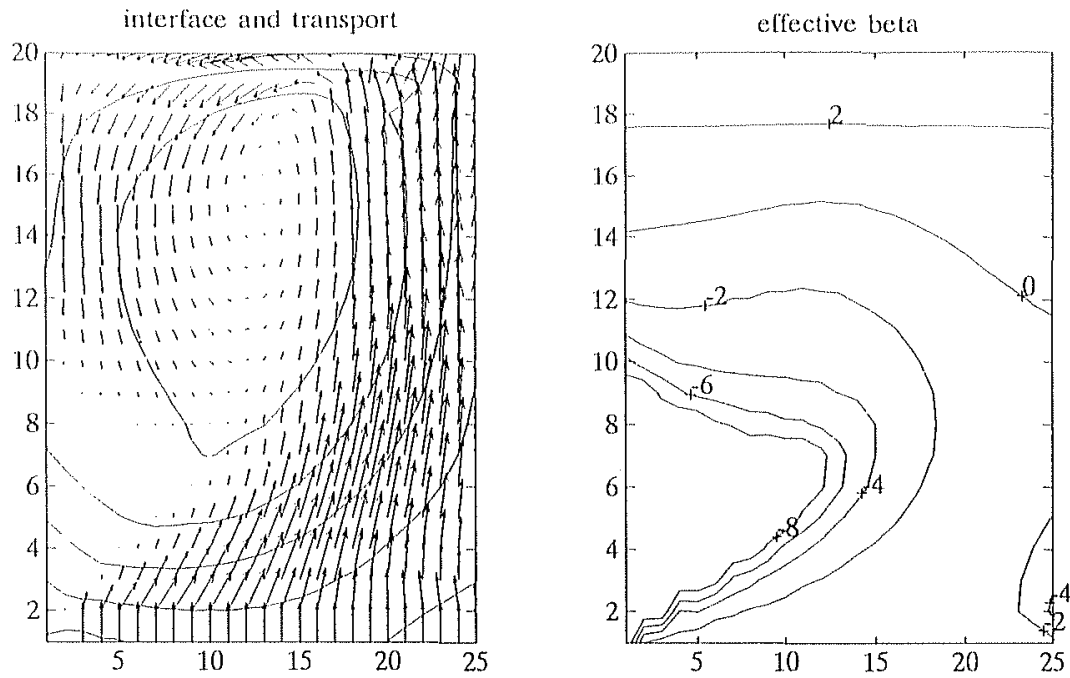

interface $\min =-871 \max =91$
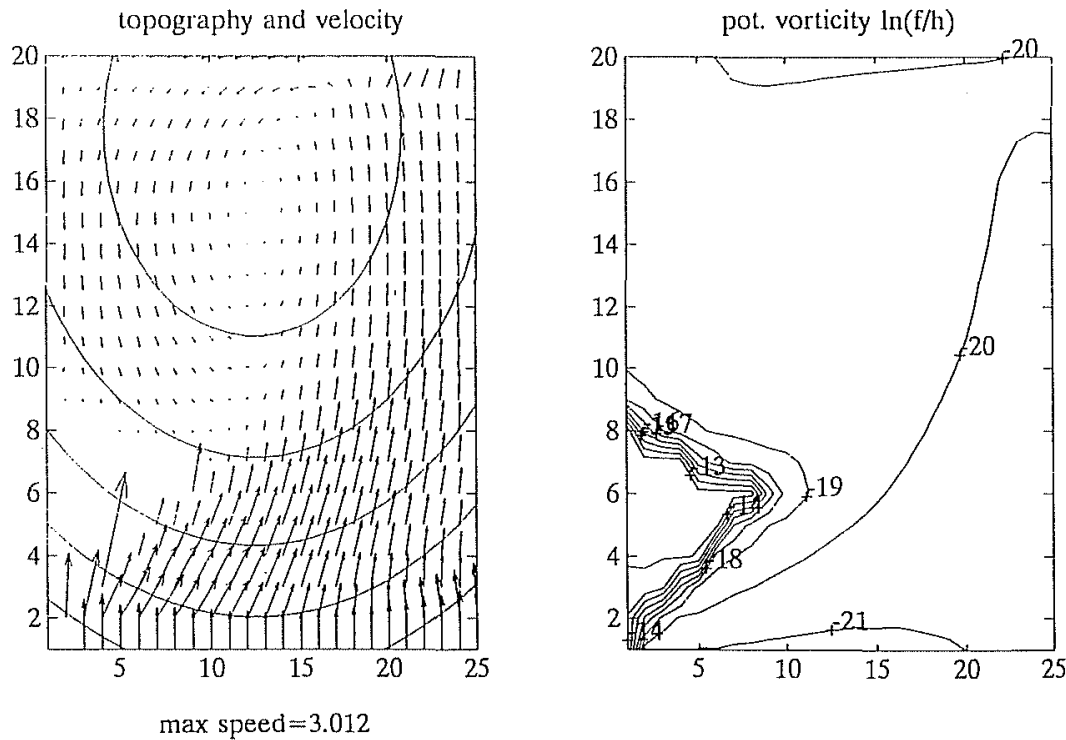

Fig. 5a. Topographic bowl case, thin layer.

effective $\beta$ there, itself a function of the solution, since $h$ appears in it. A varying thickness of the bottom layer, and thus the average effective $\beta$ in the south, changes the boundary layer width scale there.

The effective beta differs from the meridional gradient of potential vorticity $q_{y}=h^{-1}\left(\beta_{e f f}-f \eta_{y} / h\right)$ by a term which represents interface depth variations resulting from zonal geostrophic flow. In models linearized about a flat interface at a fixed depth, this difference does not exist; in this model meridional extrema of potential vorticity are not necessarily aligned with zero effective $\beta$. However, the difference is only noticeable near the source, where layer thickness variations are greatest.

In order to combine both the topographic $\beta$ effect and the hypsometric effect (variation of upwelling area enclosed by isobaths) in a single simulation, a partial bowllike shape for bottom depth was used:

$$
b=(x-\hat{x})^{2}+(y-\hat{y})^{2},
$$

where $\hat{x}, \hat{y}$ are near the center of the basin. With this choice the meridional slope $b_{y}$ varies with latitude, hence so does its role in the effective beta. The variation of area with depth is meant crudely to represent the true hypsometry of the western North Atlantic [e.g., Levitus, 1982]. Two cases will be presented, the first has a bottom layer roughly $1000 \mathrm{~m}$ thick, while in the second the upwelling has been increased to produce a thinner layer with grounding.

The interior flow pattern for the first case in which the forcing is weak enough to allow a thick layer (Figure 4; Table 1 , run $d$ ) is quite different from the flat-bottom reference. The northward flowing western boundary current from the source has disappeared because the additional stretching induced by the bottom slope forces the interior flow to absorb it. In terms of characteristics of (8) without dissipa- 

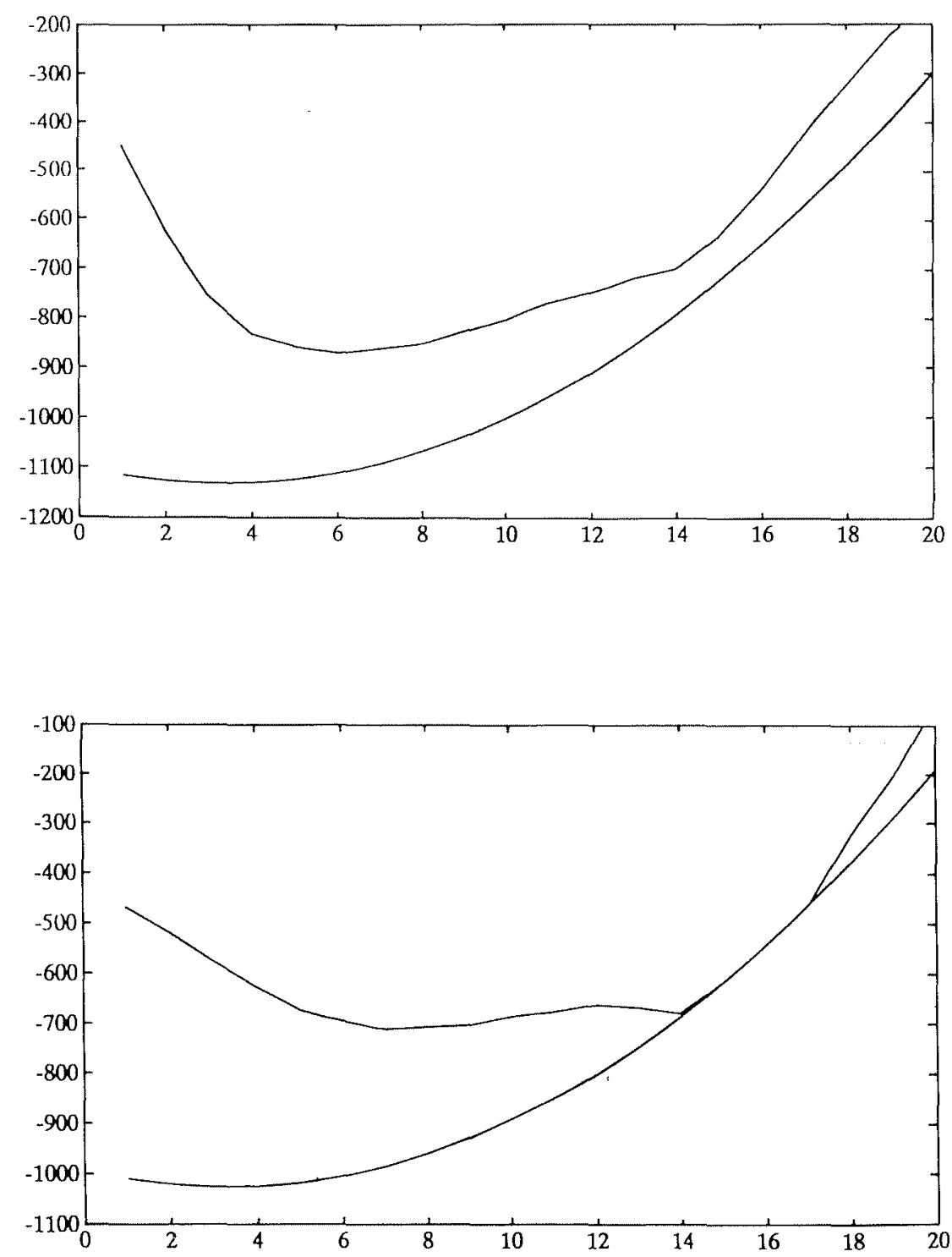

Fig. $5 b$. Two meridional sections of $h$, at $x=12$ and $x=6$. The reduced gravity $g^{\prime}=0.1 \mathrm{~cm} \mathrm{~s}^{-1}$, and $r=$ $0.3 \mathrm{~cm} \mathrm{~s}^{-1}$.

tion or time dependence, $b_{y}$ slows the westward penetration and $b_{x}$ forces characteristics to take a roundabout path to the west. Both tendencies cause a greater pressure drop in the interior, by allowing forcing more time to act, and therefore greater interior transport. The depression of the interface is strong enough along long paths reaching the southwest corner that a thinner layer there magnifies the topographic $\beta$ effect (12) and $\beta_{e f f}$ is negative. Elsewhere, the effective beta is still positive, but weak, and the recirculation cell is pulled down to a much lower latitude.

Grounding may also occur in the bowllike geometry, when upwelling is increased (Figure 5; Table 1, run $e$ ). This time, however, the outcrop area is in the southwest corner, not the northwest corner. The reason for this is that the northwest corner is now one of the deepest parts of the basin, and the combination of intensified interior flow (i.e., strong shear and strong isopycnal tilt) together with a thin layer in the southwest corner produces grounding there first. With grounding the flow concentrates in the east, as before, but no longer feeds an isolated boundary current at the northern end. Instead the flow more or less follows isobaths in a broad cyclonic gyre.

With a thin layer the topographic $\beta$ is strong enough to overcome planetary $\beta$ in the southern two-thirds of the basin. The potential vorticity structure is dominated by the outcrop. Elsewhere the distribution has a variation of the same order as previous nongrounding examples. The ridge of high $q$ between the outcrop region and the northeast corner shows that both the northward and southward moving interior flow gains potential vorticity along fluid column trajectories. A weak dissipative loss occurs over part of the westward flow along the northern boundary. On the westernmost part of the northern boundary, the bottom shoals, thickness decreases ( $q$ increases), and the flow turns south in a broad current spread out over the bottom slope.

\section{DisCussion}

A relatively simple numerical model has been used to combine the effects of strong layer thickness variation with 


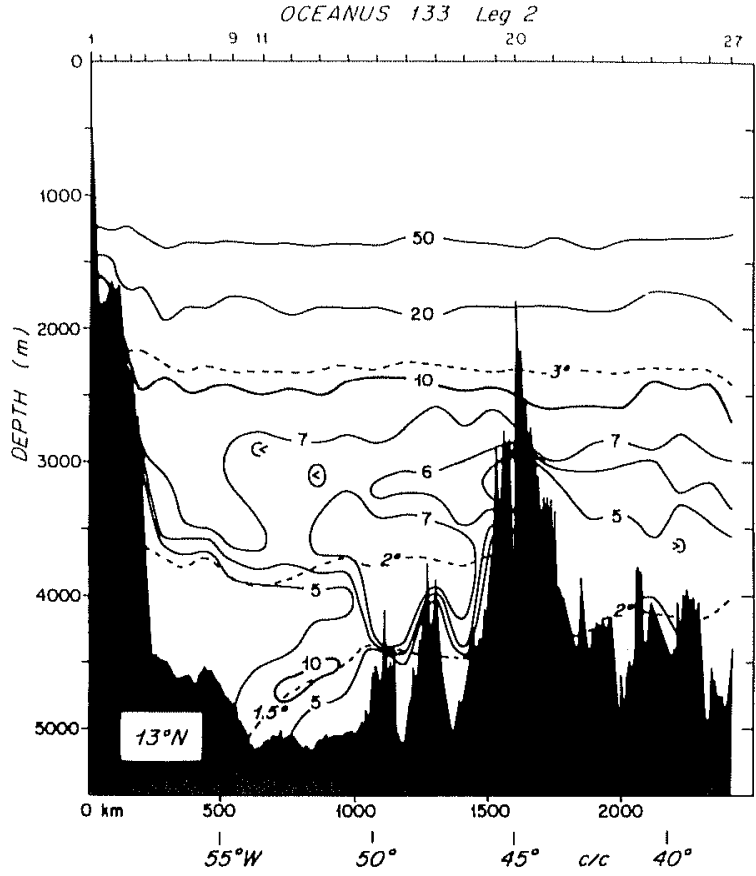

Fig. 6. Stability at $13^{\circ} \mathrm{N}\left(-\rho_{z} / \rho_{0}\right.$, units of $\left.\mathrm{cm}^{-1} \times 10^{10}\right)$. Selected isotherms have been added (dashed, ${ }^{\circ} \mathrm{C}$ ). The high stability between 4500- and 5000-m depth marks the upper limit of bottom water, near $1.5^{\circ} \mathrm{C}$; this limit is not sharply defined partly because of smoothing and partly because of actual mixing. The region of low stability in the west and above the cap is Lower North Atlantic Deep Water.

topography, in a bottom layer driven by a mass source with upwelling. Interface area and bottom layer volume may increase or decrease in response to source strength (upwelling) changes, depending on a competition between isopycnal tilt driven by upwelling and the flattening effect of long waves guided by topography and damped by friction. The upwelling was chosen to be uniform for simplicity, though more sophisticated choices are possible, including parameterizations depending on flow and thickness. In the presence of bottom topography, layer thickness variations enhance or reduce the topographic $\beta$ effect. Thus there is a feedback between the topography and flow which controls the basic structure of the bottom layer, including the direction of interior flow and the existence of boundary layers.

Three instances of eastward intensification have been illustrated here, flat-bottom grounding, topographic $\beta$ effect, and grounding in a bowllike topography. How is a choice to be made of the most likely explanation of the observed eastward transposition in the western North Atlantic? Three aspects of the circulation solutions shown here differ dramatically from one transposed flow case to another. These are the location of the outcrop region, the low-latitude western boundary current, and the distribution of potential vorticity.

Neither the case with a uniform slope in the southern half of the basin nor the bowllike topography case show a western boundary current at very low latitudes. Observations of flow at low latitude should therefore either rule in or rule out these solutions. The hydrographic measurements of Whitehead and Worthington [1982] show that at low latitudes the coldest bottom water is on the western side of the basin. This suggests that the basic grounding effect (run $b$ ) obtained with a flat bottom may be relevant and sufficient. It seems, however, improbable that the shape of the basin and the slope of the bottom play no role, but a finer resolution of flow, especially at low-latitude, is needed to make a useful comparison. Furthermore, it should be kept in mind that the time dependence of bottom flow observed by Whitehead and

ATLANTIS II 109

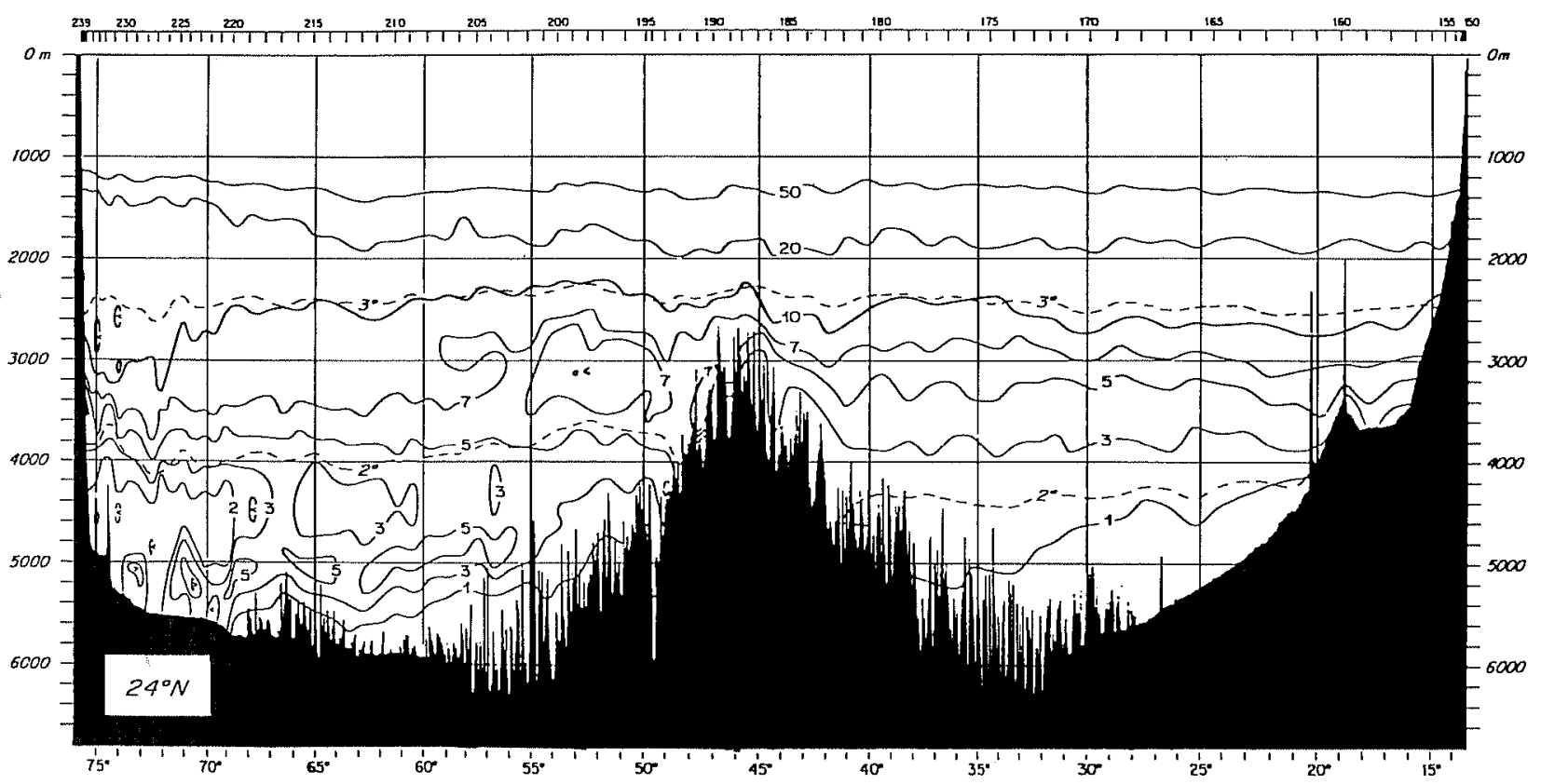

Fig, 7. Stability at $24^{\circ} \mathrm{N}\left(-\rho_{7} / \rho_{0}, \mathrm{~cm}^{-1} \times 10^{10}\right)$, with selected isotherms (dashed, ${ }^{\circ} \mathrm{C}$ ). A stratum with high stability slopes down to the west from 4500 - to 5000 -m depth, separating lower stability bottom water and Lower North Atlantic Deep Water on either side. 
Worthington [1982] could have an important role in the ocean but has no counterpart in the model described here.

Mapping the distribution of potential vorticity will help to highlight the outcrop region, as well as show the interior distribution. It varies greatly in the slope case but hardly at all throughout the interior in the two grounding cases.

In the continuously stratified ocean, two quantities have been used to make maps of potential vorticity: $f / \Delta z$, where $\Delta z$ is the distance between two isopycnals, and $f \rho_{z}$. A subjective decision is usually made regarding the degree of smoothing, which may have important consequences near the bottom in rough topography. Plots of stability $\left(-\rho_{z} / \rho_{0}\right)$ prepared in the context of a separate study [Speer, 1988] show promise for the mapping of potential vorticity $\left(f \rho_{z}\right)$ in the bottom layer. In sections at $13^{\circ} \mathrm{N}$ and $24^{\circ} \mathrm{N}$ (Figures 6 and 7) the bottom layer is seen to be capped by a region of high stability. The magnitude of the stability in and near the maximum decreases by half from $13^{\circ} \mathrm{N}$ to $24^{\circ} \mathrm{N}$, roughly in proportion with $f$, and at both latitudes the high-stability cap tilts down to intersect the bottom, east of where it would otherwise run into the western boundary. Maps of both stability and potential vorticity may help to define interior structure and an interface for what in the ocean is a stratified bottom water mass, not a homogenous layer.

A natural step to take with the model is to parameterize the upwelling. Using a simple Richardson number criterion tended to turn on upwelling everywhere or, on the contrary, shut it off everywhere. Finer distinctions between upwelling regimes probably require a model with more physics.

\section{Appendix: Numerical Model}

For the purpose of the numerical solution, it is convenient to solve for the transports rather than for the velocities, so we define

$$
U=u h \quad V=v h
$$

The model equations (1), (2), and (4) can then be used to derive the following set of equations:

$$
\eta \equiv b+h
$$

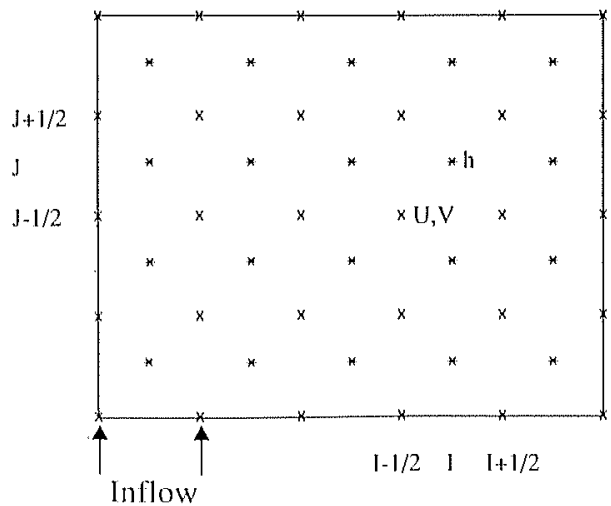

Fig. Al. The staggered finite difference grid used for the numerical solution of our model equations. $U, V$ points are indicated by crosses, and $h, b, \eta$ points are indicated by stars. Note that only $U$, $V$ points occur on the boundaries, where the transports are specified as described in the text. The thickness $h$ occurs only in the interior of the grid, where it is calculated using the model equations.

$$
\begin{gathered}
U=\frac{-g^{\prime} h}{f^{2}+(r / h)^{2}}\left(f \eta_{y}+r \eta_{x} / h\right) \\
V=\frac{-g^{\prime} h}{f^{2}+(r / h)^{2}}\left(-f \eta_{x}+r \eta_{y} / h\right) \\
h_{t}=-\left(w_{e}+U_{x}+V_{y}\right)
\end{gathered}
$$

These equations are solved on the staggered grid shown in Figure A1. In finite difference form, the equation for the thickness $h$ is solved using centered differencing on the staggered grid and an Adams-Bashforth time stepping algorithm. First, the transports are evaluated as follows. Define

$$
\begin{aligned}
& \bar{h}^{n} \equiv\left(h_{i, j}^{n}+h_{i-1, j}^{n}+h_{i, j-1}^{n}+h_{i-1, j-1}^{n}\right) / 4, \\
& \eta_{x}^{n} \equiv\left(\eta_{i, j}^{n}+\eta_{i, j-1}^{n}-\eta_{i-1, j}^{n}-\eta_{i-1, j-1}^{n}\right) / 2 \Delta x, \\
& \eta_{y}^{n} \equiv\left(\eta_{i, j}^{n}+\eta_{i-1, j}^{n}-\eta_{i, j-1}^{n}-\eta_{i-1, j-1}^{n}\right) / 2 \Delta y,
\end{aligned}
$$

giving the transports

$$
U_{i-1 / 2, j-1 / 2}^{n}=-\frac{g^{\prime} \bar{h}^{n}}{f_{j-1 / 2}^{2}+\left(r / \bar{h}^{n}\right)^{2}}\left(f_{j-1 / 2} \eta_{y}^{n}+r \eta_{.}^{n} / \bar{h}^{n}\right),
$$

$$
V_{i-1 / 2, j-1 / 2}^{n}=-\frac{g^{\prime} \bar{h}^{n}}{f_{j-1 / 2}^{2}+\left(r / \bar{h}^{n}\right)^{2}}\left(-f_{j-1 / 2} \eta_{x}^{n}+r \eta_{y}^{n} / \bar{h}^{n}\right)
$$

Next, the time rate of change is evaluated and the equation for the thickness is stepped forward in time:

$$
\begin{aligned}
h_{t, i j}^{n}= & -\left[w_{e, i j}+\left(U_{i+1 / 2, j-1 / 2}^{n}\right.\right. \\
& \left.-U_{i-1 / 2, j-1 / 2}^{n}-U_{i-1 / 2, j+1 / 2}^{n}\right) / 2 \Delta x \\
& +\left(V_{i-1 / 2, j+1 / 2}^{n}+V_{i+1 / 2, j+1 / 2}^{n}\right. \\
& \left.\left.-V_{i-1 / 2, j-1 / 2}^{n}-V_{i+1 / 2, j-1 / 2}^{n}\right) / 2 \Delta y\right] \\
& h_{i j}^{n+1}=h_{i j}^{n}+\Delta t\left[\frac{3}{2} h_{t, i j}^{n}-\frac{1}{2} h_{i, i j}^{n-1}\right],
\end{aligned}
$$

where $\Delta x$ and $\Delta y$ are the distances between $h$ grid points in the $x$ and $y$ directions. Both transports $(U, V)$ are set to zero on the solid boundaries. At the inflow, on the southern boundary, the normal transport $(V)$ is specified, while the tangential transport $(U)$ is set to zero.

A no-slip boundary condition is used for the velocity field at the boundaries. Such a condition is, in fact, not supported by the Rayleigh friction used in the above momentum equations, but may be regarded as a subgrid parameterization of mixing processes near the boundaries that are not explicitly modeled.

At every time step the thickness field $h_{i, j}^{h}$ is scanned, and where the thickness calculated using the above time stepping procedure is zero or negative, it is replaced by a small number $\left(10^{-15} \mathrm{~cm}\right)$. This, through the frictional terms that are proportional to $r / h$, effectively reduces the velocities at these grid points to zero, while preventing division by zero during the model run. 
Acknowledgments. K.G.S. has been supported by the Bundesministerium für Forschung und Technologie and the Centre National de la Recherche Scientifique. A reviewer's thoughtful questioning led to improvements. Hydrographic data for the stability plots were originally collected by M. McCartney $\left(13^{\circ} \mathrm{N}\right)$, and C. Wunsch, D. Roemmich, and M. McCartney $\left(24^{\circ} \mathrm{N}\right)$. Department of Environmental Sciences and Energy Research of the Weizmann Institute of Science contribution 55.

\section{REFERENCES}

Colin de verdiere, A., Buoyancy-driven planetary flows, I. Mar. Res., 46, 215-265, 1988.

Cushman-Roisin, B., Frontal geostrophic dynamics, J. Phys. Oceanogr., 16, 132-143, 1986.

Huang, R. X., The thermocline and current structure in subtropical/ stibpolar basins, Ph.D. dissertation, 178 pp., Mass. Inst. of Technol./Woods Hole Oceanogr. Inst., Woods Hole, Mass., 1984.

Kawase, M., Establishment of deep ocenn circulation driven by deep-water production, J. Phys. Oceanogr., 17, 2294-2317, 1987.

Kawase, M., and D. Straub, Spinup of a source-driven circulation in an abyssal basin in the presence of bottom topography, J. Phys. Oceanogr., 21, 1501-1514, 1991.

Levitus, S., Climatological atlas of the world ocean, Prof. Pap. 13, $173+x v$ pp., Natl. Oceanic and Atmos. Admin., Rockville, Md., 1982.

Mantyla, A. W., and I. L. Reid, Abyssal characteristics of the World Ocean waters, Deep Sea Res., 30, 805-833, 1983.

Nof, D., N. Paldor, and S. VanGorder, Abyssal gyres, Geophys. Astrophys. Fluid Dyn., 58, 173-196, 1990.

Rhines, P. B., Deep planetary circulation and topography: Simple models of midocean flows, J. Phys. Oceanogr., 19, 1449-1470, 1989.

Rhines, P. B., and P. M. McCready, Boundary control over the large-scale circulation, in Parameterization of Small-Scale Processes, edited by P. Mueller, pp. 75-99, 'Aha Huliko'a workshop, Hawaii Institute of Geophysics special publication, University of Hawaii, Honolulu, 1989.

Salmon, R., A simplified linear ocean circulation theory, J. Mar. Res., 44, 695-711, 1986.

Smith, P. C., A streamtube model for bottom boundary currents in the ocean, Deep Sea Res., 22, 853-874, 1975.
Speer, K. G., The influence of geothermal sources on deep temperature, sallinity, and flow fields, Ph.D thesis, 148 pp., Mass. Inst. of Technol./Woods Hole Oceanogr. Inst., Woods Hole, Mass., 1988.

Speer, K., and M. S. McCartney, Bottom water flow in the western North Atlantic, J. Phys. Oceanogr., 22, 83-92, 1992.

Speer, K. G., and E. Tziperman, Convection lirom a source in an ocean basin, Deep Sea Res., 37, 431-446, 1990.

Stommel, H., and A. B. Arons, On the abyssal circulation of the world ocean, I, Stationary planetary flow patterns on a sphere, Deep Sea Res., 6, 140-154, 1960.

Straub, D., and P. B. Rhines, Effects of large-scale topography on abyssal circulation, J. Mar. Res., 48, 223-253, 1990.

Tziperman, E., On the role of interior mixing and air-sea fluxes in cletermining the stratification and circulation of the oceans, $J$. Phys. Oceamogr., 16, 680-693, 1986.

Warren, B. A., Deep circulation of the world ocean, in Evolution of Physical Oceanography, Scientific Surveys in Honor of Henty Stommel, edited by B. A. Warren and C. Wunsch, pp. 6-41, MIT Press, Cambridge, Mass., 1981.

Warren, B. A., The deep water of the Central Indian Basin, J. Mar. Res., 40, suppl., 823-860, 1982.

Warren, B. A., and W. B. Owens, Some preliminary results concerning deep northern-boundary currents in the North Pacific, Prog. Oceanogr., 14, 537-551, 1985.

Warren, B. A., and K. G. Speer, Deep circulation in the eastern South Atlantic Ocean, Deep Sea Res., 38, suppl., 281-322, 1991.

Whitehead, J. A., and L. V. Worthington, The flux and mixing rates of Antarctic Bottom Water within the North Atlantic, $J$. Geophys. Res., 87, 7903-7924, 1982.

Y. Feliks, Institute for Biological Research, Nes-Ziona 76100 , Israel.

K. Speer, Laboratoire de Physique des Océans, IFREMER, B. P. 70, 29280 Plouzané, France.

E. Tziperman, Weizmann Institute of Science, Rehovot 76100, Israel.
(Received February 4, 1992; revised October 28, 1992; accepted November 4, 1992.) 